\title{
Further avoidance studies with goldfish: Brightness discrimination and transposition
}

\author{
D. J. ZERBOLIO, JR. \\ University of Missouri, St. Louis, Missouri
}

\begin{abstract}
In two experiments using the shuttlebox apparatus and negative (shock) reinforcement, goldfish learned to discriminate between same color signals differing only in brightness and to choose the brightness level paired with shock-omission to avoid shock. Experiment 1 compared two signal presentation procedures, one presenting only one signal at a time but allowing for successive alternation of signals within a trial (SUC) and the other presenting both signals simultaneously (SIM). SIM performance was superior to SUC in acquisition and showed more positive transfer with signal color changed (CC), more negative transfer with brightness-level-shock pairing reversed $(R)$, and, although attenuated, more negative transfer with both signal color changed and shock pairing reversed $(C C+R)$. Further, SIM relearning following reversal was faster than SUC. In Experiment 2, using the SIM procedure, eight transfer groups were tested for transposition with one of their original acquisition signals replaced in transfer by a signal bearing either the same or the opposite brightness relationship to the retained signal. Four transfer groups showed positive and four showed negative transfer effects, but all eight groups showed transfer performances consistent with a transpositional expectation. The present two experiments alone do not elucidate the mechanisms accounting for either the SIM superiority over SUC in Experiment 1 or the transposition effect in Experiment 2. These experiments show only that the transposition phenomenon can be supported by negative reinforcement procedures, which has not been demon. strated before. Now that techniques exist for producing negatively reinforced transposition, work investigating the underlying mechanisms and comparing them with the mechanisms of positively reinforced transposition can begin.
\end{abstract}

Using the newly developed linear presentation procedure (LPP), Zerbolio (1981) and Zerbolio and Royalty (1982) showed that goldfish could learn to choose between different color signals to avoid shock in the shuttlebox apparatus. Furthermore, since the LPP supports transfer testing, Zerbolio (1981) demonstrated a reversal learning effect when signal-shock pairing was reversed, and Zerbolio and Royalty (1982) showed that goldfish could learn both to avoid the shock-paired signal and to approach the shock-omission-paired signal. The latter two findings occurred when, in transfer, goldfish were made to choose between the original shock-paired signal and a new neutral signal, or between the original shock-omission-paired signal and a new neutral signal. In the first case, goldfish chose the new neutral signal, and in the second, they continued to choose the original shock-omission-paired (safe) signal. However, the utility of the LPP may be limited because, in principle, color discriminations are fairly easy, and the LPP is essentially a "within-trials successive presentation" technique.

Reasons for suspecting a limited usefulness come from the positive reinforcement literature. The acquisition of

The author wishes to thank David R. Holtgrave, now at the University of Illinois, for his initial interest in this project, and for his substantial help in running animals, collecting data, and analyzing the results.

The author's mailing address is: Department of Psychology, University of Missouri-St. Louis, 8001 Natural Bridge Rd., St. Louis, MO 63121 . more difficult discriminations, as well as of the more interesting transfer phenomena, such as transposition (which may involve learning relationships), is facilitated much more by simultaneous-than by successive-presentation procedures (Bitterman, Tyler, \& Elam, 1955; Gonzalez \& Shepp, 1961; MacCaslin, 1954; Marsh, 1967; Riley, 1968; Sachs, 1969; Spence, 1952). Thus, the successivepresentation characteristics of LPP may limit its use as a tool for investigating discrimination learning and transfer effects. To fully appreciate the successive-presentation characteristics of the LPP, one must carefully scrutinize the procedure.

In the LPP, the intertrial interval (ITI) is indicated by white illumination and the onset of a trial is signaled by the offset of white light, rendering the shuttlebox dark. Although there are two other different color signals that represent the available choices, they are not shown until the initial response of a trial, and then only one at a time. Specifically, the shuttlebox remains dark until the goldfish's first response of the trial; if no response occurs, shock is delivered as scheduled. Given this first response, one or the other, but not both, of the color signals is presented (the same color at both ends of the tank). The goldfish can control prevailing color by additional shuttling, because each additional response causes the two color-choice signals to alternate. Since the color signal presented contingent on the first response is determined randomly, avoidance is not guaranteed by responding 
once, or a random number of times per trial, because each response alternates the prevailing color. To avoid at better than $50 \%$, the goldfish must initially respond to gain access to the (randomly determined) first alternative signal and, if that signal is the shock-paired signal, must respond again to alternate the signal state to the shockomission-paired signal. Clearly, the choice mechanism involves controlling the signal state of the entire shuttlebox via the response-contingent signal-alternation programming. Because of the alternation mechanism, the animal never sees more than one choice alternative at a time, which makes it, nominally at least, a successivepresentation procedure. It is possible to modify the presentation procedure to enable the simultaneous presentation of both signals in the shuttlebox apparatus.

Suppose that, as in the LPP, the ITI is indicated by white light, trial onset is signaled by dark, neither of the choice alternatives is presented until the first response occurs, and, if no response occurs, shock is delivered as scheduled. Further, suppose the first response turns on both alternative choice signals, one at each end of the shuttlebox. Additional responses cause no change in these signals, except, of course, to transport the goldfish from one end (and signal) to the other end (and other signal). Since the end displaying a specific choice alternative would be varied randomly from trial to trial, a single response or random responding would guarantee no more than $50 \%$ avoidance. To avoid at better than $50 \%$, the goldfish would have to initially respond to gain access to both signals (one at each end of the apparatus) and then position itself (by shuttling) at the end displaying the preferred color signal. Since both signals are present at the same time, the procedure is, nominally at least, a simultaneouspresentation procedure.

Although a Y-maze simultaneous-presentation avoidance-task has been devised for goldfish (Zerbolio \& Wickstra, 1979, 1980), the development of a simultaneous-presentation avoidance task for the shuttlebox would be desirable simply because the bulk of the current goldfish shock-avoidance choice discrimination and transfer results have been obtained in the shuttlebox apparatus. It would also be desirable to develop signals differing in brightness rathei than color to facilitate comparisons with the earlier literature, because many earlier discrimination learning studies used animals without color vision (e.g., rats), and many of the earlier demonstrations of transposition used some form of brightness discrimination (e.g., Kohler, 1918/1939; Lawrence \& DeRivera, 1954; Riley, 1958), including demonstrations of transposition by goldfish (Perkins, 1931; Perkins \& Wheeler, 1930). However, it is important to remember that all of the earlier transposition demonstrations used positive reinforcement techniques. As far as is known to the author, transposition has never been demionstrated with negative or shock avoidance reinforcement procedures. The first step toward demonstrating negatively reinforced transposition is to develop appropriate signals and appropriate training and transfer procedures. Experiment 1 was designed toward achieving this end.

\section{EXPERIMENT 1}

Experiment 1 had two goals-to determine if goldfish could learn to avoid shock by choosing between two signals differing only in brightness (same color), and to compare the within-trials successive-presentation format of the LPP with the proposed simultaneous-presentation procedure in the shuttlebox apparatus. Both original learning and transfer comparisons were planned. Following original learning, three transfer comparisons were made. The first involved changing the color of the signals (CC) without changing the brightness-level-shock pairing; the second involved reversing $(R)$ the brightness-level-shock pairing without changing the signal color; and the third involved changing the signal color and reversing $(C C \pm R)$ the brightness-level-shock pairing. If there were any differences between the successive- (LPP) and simultaneous-presentation formats in the shuttlebox, one would expect goldfish to learn something about the relationships between signals in the simultaneous format, but not in the LPP format. This would be expected to produce faster original learning and larger positive and negative transfer effects.

\begin{abstract}
Method
Subjects

Forty-eight 5 to 6-cm-long goldfish, obtained from Ozark Fisheries, served as subjects. All were housed in individual $7.5 \times 11.4$ $\mathrm{x} 12.5 \mathrm{~cm}$ deep holding tanks. Water in the holding tanks was well filtered and aerated at all times. In addition, to remove heavier debris, $25 \%$ of the water in the holding tanks was siphoned off daily and replaced with fresh water. All holding tanks were emptied, scrubbed, and refilled with fresh water weekly. Temperature $\left(21^{\circ} \pm .1^{\circ} \mathrm{C}\right)$ and $\mathrm{pH}(7 \pm .1)$ were held constant throughout the experiment.
\end{abstract}

\section{Apparatus}

Four identical $29.2 \times 11.4 \times 11.4 \mathrm{~cm}$ deep shuttleboxes were used. Each shuttlebox contained a single centered $6.35-\mathrm{cm}$-high hurdle that had a $9-\mathrm{cm}$ flat top and ramps that sloped $45^{\circ}$ into deep wells on each end. Water was maintained at $2 \mathrm{~cm}$ over the flat top of the hurdle at all times. The water was constantly aerated by end inserts, which were sandblasted to diffuse illumination. To remove debris, $25 \%$ of the water was removed each day by siphoning and replaced with fresh water. Weekly, the shuttleboxes were emptied, cleaned, and refilled with fresh water. Shuttle activity was monitored via photocells focused across the ends of the 9-cm flat top of the hurdle. Photocell light sources were $2.5-\mathrm{V}$ dc prefocused penlight bulbs, operated at $1.5 \mathrm{~V}$ ac to extend bulb life. During running, each shuttlebox was covered with a clear plastic lid.

Different light signals were provided by three fixtures, one located at each end of the shuttleboxes and one located on top of the shuttlebox lid. ITI illumination, which cast light directly down into the shuttleboxes, was provided by white $7-\mathrm{W} 110$-V ac Christmas tree bulbs in fixtures placed on the clear plastic lids. Responsecontingent colored illlumination, which cast light directly on the end walls, was provided by $7-\mathrm{W} 110-\mathrm{V}$ ac Christmas tree bulbs in fixtures located at each end of the shuttleboxes. The two responsecontingent colors used were red $(620 \pm 4 \mathrm{~m} \mu)$ and green (517 $\pm 2 \mathrm{~m} \mu$ ). Brightnesses were measured using a Minolta Spotmeter $\mathrm{M}$, positioned $15 \mathrm{~cm}$ above the shuttlebox. Using a mirror, the measurements were made through $15 \mathrm{~cm}$ of water for the end lamps and through $4 \mathrm{~cm}$ of water for the ITI lamp. Two brightnesses, low $(\mathrm{L})$ and high $(\mathrm{H})$, were used for each color: red $\mathrm{L}$ $(.8 \pm .2 \mathrm{~mL})$ and red $\mathrm{H}(6.3 \pm .2 \mathrm{~mL})$ brightnesses and green $\mathrm{L}$ 
$(.2 \pm .01 \mathrm{~mL})$ and green $\mathrm{H}(5.1 \pm .4 \mathrm{~mL})$ brightnesses. In addition, translucent plastic plates were placed between each end light fixture and the end wall of the shuttlebox to diffuse illumination. Overhead illumination luminances were $7.2 \pm 1.2 \mathrm{~mL}$. Opaque black plastic plates were positioned inside the hurdles across the shuttlebox from one side to the other; these served to visually isolate the ends so that goldfish at the bottom of the deep well at one end could not see the color of the illumination at the other end. However, if the goldfish swam to the surface or to the top of the hurdle, both end colors were visible. Shock, in $200-\mathrm{msec}$ pulses at $7-\mathrm{V}$ ac $(.69 \mathrm{~V} / \mathrm{cm})$, was delivered by $28 \times 10.2 \mathrm{~cm} \mathrm{22-ga} \mathrm{stainless} \mathrm{steel}$ plates affixed to the interior side walls of each shuttlebox.

\section{Procedure}

The experiment was divided into a 15-day original acquisition phase and an additional 15-day transfer phase, for 30 total days. All animals were run 40 trials a day with a variable 60 -sec interval (ranging from 30 to $90 \mathrm{sec}$ ) separating trials. The goldfish were run 5 days a week and allowed to rest on weekends. White overhead illumination indicated the ITI. At the start of each trial, the white overhead light was extinguished, rendering the shuttleboxes dark. Regardless of the presentation procedure, the shuttlebox remained dark until the animal's first response or until the trial ended. Each trial consisted of a 13.2-sec period, starting with the termination of the overhead white ITI illumination. The first $10 \mathrm{sec}$ were shock-free; shocks were scheduled at 10,12, and $13 \mathrm{sec}$. The shocks could be avoided (omitted) only if the goldfish met the proper criterion at each of the scheduled shock times. A response constituted swimming from the end currently occupied (and the photobeam last broken) to the other end, through the other photobeam. Because of the shallow $(2 \mathrm{~cm})$ depth there, goldfish rarely remained on the top of the hurdle. If the goldfish did not respond within the first $10 \mathrm{sec}$, the first shock was delivered. If a shuttle response was elicited by the first shock, and the animal's response produced the proper criterion, further shocks were omitted (or escaped). To be credited with an avoidance, all three shocks on a trial had to be omitted. The purpose of the three-shock procedure was to avoid crediting a randomly respnding animal with avoidances (see Zerbolio \& Royalty, 1983). At $13.2 \mathrm{sec}$, all trial illumination was extinguished and the white ITI illumination was reestablished. Regardless of the procedure, no brightness signals were presented until the goldfish's initial response of the trial. These procedures were common to all phases of the experiment, including both original learning and transfer testing.

Original learning. Half of all the animals had their responsecontingent signals presented successively (LPP); the remainder had their response-contingent signals presented simultaneously.

Successive presentation (SUC): Half $(n=24)$ of the goldfish were trained with the LPP procedure. In this procedure, white light indicated the ITI and termination of the ITI or dark signaled trial onset; if no response occurred, the shuttlebox remained dark and shock was delivered as scheduled. The alternative brightness-choice signals were not presented until after the initial response of a trial had been made. The initial response of the trial turned on one or the other, but not both, of the brightness signals ( $\mathrm{H}$ or $\mathrm{L}$ ) at both ends of the shuttlebox; additional responses caused the prevailing signal to alternate with response. The brightness signal ( $\mathrm{H}$ or $\mathrm{L})$, which turned on, contingent upon the first response, was randomly determined. If, at scheduled shock times, the prevailing signal was anything but the signal paired with shock omission ( $\mathrm{H}$ or $\mathrm{L}$ ), shock was delivered as programmed. If the prevailing signal was the shockomission-paired signal during all three scheduled shock times, an avoidance was recorded.

Simultaneous presentation $(S I M)$. Half $(n=24)$ of the goldfish were trained with a simultaneous-presentation procedure. As in LPP, white light indicated the ITI, dark signaled trial onset, the alternative choice signals were not presented until the first response had been made, and, if a response did not occur, the shuttlebox remained dark and shock was delivered as scheduled. The goldfish's first response turned on both brightness signals ( $\mathrm{H}$ and $\mathrm{L}$ ), one at each end of the shuttlebox. No further signal changes occurred following the initial response of the trial. The end showing a specific choice signal (e.g., H) varied randomly from trial to trial. In order to avoid shock, the goldfish had to initially respond prior to the first scheduled shock at $10 \mathrm{sec}$, determine if the signal on the end it then occupied was the appropriate (i.e., shock-omission-paired) signal, and, if not, to shuttle to the other end for the appropriate signal condition. Essentially, the goldfish had to respond initially to turn on both different brightness signals ( $\mathrm{H}$ and $\mathrm{L}$ ) at the ends of the shuttlebox, and then choose, on the basis of displayed brightness signal, which end of the shuttlebox to occupy on each trial. If the animal occupied the end showing the shock-omission signal at all three scheduled shock times, an avoidance was recorded. If the animal failed to respond or occupied the shock-paired-signal end at any of the shock times, shock was delivered.

Both the SUC and the SIM presentation-procedure originallearning groups were balanced for signal color (half red and half green) and for which brightness level was shock-paired (half had the $L$ level paired, and the remainder had the $H$ level paired).

Transfer testing. In transfer testing, each procedure group (SUC and SIM) was divided into three equal groups of eight goldfish each. Two transfer changes were used. The first involved changing the signal colors (CC) from red to green or vice versa, and the second involved reversing the brightness-level shock pairing $(R)$ in transfer. For example, if $\mathrm{H}$ was paired with shock and $\mathrm{L}$ was paired with shock omission in acquisition (H-L+), $\mathrm{H}+\mathrm{L}-$ shock pairing was used in transfer. One-third of the SUC and SIM groups were tested in transfer with their original signal color changed (CC, red to green, or vice versa) but with their brightness-shock pairing as in original learning; one-third were tested with their original brightness-shock pairing reversed in transfer (R); and one-third were tested with both their original signal color changed and their brightness-shock pairing reversed $(C C+R)$. Except for these changes, the conditions of transfer testing were identical to those of original learning.

Learning measures. Each day, two measures were recorded for each animal: the number of trials with response (TwR) and the number of avoidances (A), or the number of TwR in which the animal missed shock (met the criterion) at all three scheduled shock times. With these data, an additional measure, the discrimination index (DI), was calculated. The DI measure is $[(\mathrm{S}+)-(\mathrm{S}-)] / 40$, where $\mathrm{S}+$ is the number of TwR in which the animal met the criterion at all three shock times and thus avoided all shocks, where $S-$ is the number of TwR in which the animal responded before shock but received at least one scheduled shock, and where S+ and Ssum to TwR. The DI measure has limits of +1.00 , where +1.00 indicates choice of the shock-omission-paired signal on all (40) trials, -1.00 indicates choice of the shock-paired signal on all trials, and 0.00 indicates chance performance regardless of the number of TwR. Furthermore, because $(S+)-(S-)$ is divided by 40 , the number of trials per day, the DI measure discriminates between animals that respond on just a few trials and those that respond on many or all trials.

\section{Results and Discussion}

The data for the original learning and transfer testing phases were separated to facilitate analysis, presentation, and discussion. The 15 days of each phase were combined into five 3-day blocks for presentation and analysis. There were no essential differences between the 15-day analyses and the five 3-day block analyses in either original learning or transfer, so only the five 3-day block analyses are presented. The data for each phase were initially analyzed using a three-way ANOVA, with SIM/SUC, and CC, R, and $C C+R$ as independent dimensions, and five 3-day blocks as a repeated dimension. When necessary, addi- 
tional partitions were done to facilitate explanation. All results are presented in Figures 1 and 2. Figure 1 shows the percentage of TwR and avoidances per 3-day (120 trials) block for the original learning and transfer phases for all conditions, and Figure 2 shows the mean DI results over the same phases and conditions. Figure 1 also shows $\mathrm{E}$ (avoidances) which is a random chance avoidance rate. $\mathrm{E}$ (avoidance) is half of the TwR rate $(.5 \times \mathrm{TwR})$ and is shown for comparison purposes only.

The left panels of Figures 1 and 2 show the original learning results for both the SIM and SUC presentationprocedure-trained goldfish. From these figures, it is clear that although both SIM and SUC groups responded on about the same number of trials (TwR, Figure 1), the SIM group learned to choose the brightness level paired with shock omission faster and more often (higher asymptote, Figure 2) than did the SUC group, and avoided on more trials (Figure 1). Also, the superiority of the SIM procedure in discriminative choice accuracy is especially evident on the DI measure (left panel, Figure 2). These impressions were completely supported by the analysis.

Both the SIM and SUC groups showed significant TwR increases in original learning $[F(4,168)=463.56, p<$ $.01]$, but no other significant effects were found in the TwR analysis. The avoidance and DI measures produced comparable patterns of results, so only the DI analysis is reported. Both SIM and SUC groups showed significant DI increases over blocks of training $[F(4,168)=$ $170.09, \mathrm{p}<.01$ ], with SIM's being significantly higher than SIC's $[F(1,42)=19.29, \mathrm{p}<.01]$; a significant interaction between SUC/SIM and blocks was also observed $[F(4,168)=6.02, p<.01]$. Partitions of this interaction found that the SIM group was significantly higher than the SUC group in DI performance on all but the first 3-day block of original learning. These differences are indicated in the left panel of Figure 2. Comparable differ-

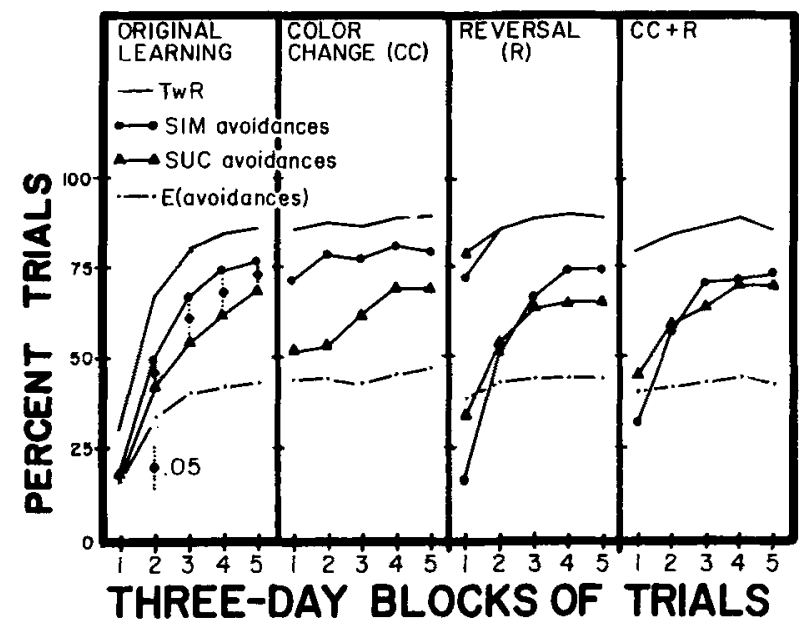

Figure 1. The mean percent 3-day-block trials with response (TwR), avoidances, and E (avoidances) for the SIM and SUC $(n=24)$ groups in original learning (panel 1), and for the SIM and SUC color change (CC), reversal ( $R$ ), and color change and reversal $(C C+R)$ transfer conditions (panels 2,3 , and 4, respectively). $\mathrm{E}$ (avoidances), which is $.5 \times \mathrm{TwR}$, is included for comparative purposes only.

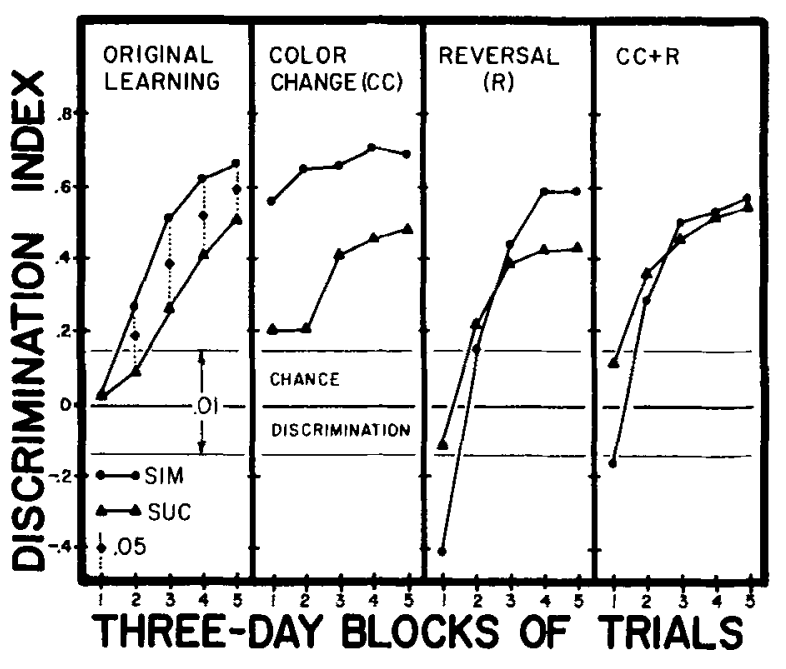

Figure 2. The mean 3-day-block discrimination indexes (DI) for the SIM and SUC groups in original learning (panel 1), and for the SIM and SUC color change (CC), reversal (R), and color change and reversal $(C C+R)$ transfer conditions (panels 2,3 , and 4, respectively).

ences on the avoidance measure are indicated in Figure 1.

The transfer results are equally clear. Note that with only the signal color changed (CC), the SIM group showed significantly more positive transfer than the SUC group (second panels of Figures 1 and 2). When the signal shock pairing was reversed $(R)$, the SIM group showed significantly more negative transfer than the SUC group in the initial phases of transfer testing, but recovered faster and again reached a higher asymptote by the end of transfer testing (third panels of Figures 1 and 2). The last transfer procedure with both the original learning color changed and the signal-shock pairing reversed $(C C+R)$ showed an attenuation of the reversal effect for both SIM and SUC seen in the R procedure alone, but that the SIM group still showed more negative transfer than did the SUC group (right panels of Figures 1 and 2). All of these impressions were confirmed by analysis of the transfer results.

For the CC groups, no differences between SIM and SUC or change over blocks of transfer testing on the TwR measure were observed. On the DI measure, both SIM and SUC showed significant increases over blocks $[F(4,168)=6.85, p<.01]$ and the SIM group was significantly higher throughout transfer testing $[\mathrm{F}(1,42)=$ $12.54, \mathrm{p}<.01]$. No other significant effects were observed.

In transfer, the result patterns for the avoidance and DI measures were similar in all cases. Thus, for brevity, only the DI analyses are presented.

For the R groups, an increase in TwR performance over blocks of transfer testing $[\mathrm{F}(4,168)=23.39, \mathrm{p}<.01]$ and a SIM/SUC $\times$ blocks of testing interaction were observed $[F(4,168)=3.44, p<.01]$. Partitions showed that the SIM group responded on fewer trials than the SUC group only on the first 3 -day block $[F(1,210)=3.90$, $\mathrm{p}<.05]$. The DI analysis found significant increases over 
blocks $[F(4,168)=81.56, p<.01]$ and a significant SIM/SUC $\times$ blocks interaction $[F(4,168)=6.77, p<$ $.01]$. Partitions found that the SIM group was significantly lower on the DI measure than the SUC group only on the first block of transfer testing $[F(1,210)=7.34, p<.01]$.

For the $\mathrm{CC}+\mathrm{R}$ groups, no differences in TwR were observed between SIM and SUC, but both showed significant increases over blocks of transfer testing $[\mathrm{F}(4,168)$ $=9.43, \mathrm{p}<.01]$. Both SIM and SUC increased their DI performances over blocks of transfer testing $[\mathrm{F}(4,168)$ $=44.95, \mathrm{p}<.01]$, and a SIM $/ \mathrm{SUM} \times$ blocks interaction was observed $[\mathrm{F}(4,168)=3.18, \mathrm{p}<.05]$. Partitions showed the SIM group with lower DI performance than the SUC group only on the first block of transfer testing $[F(1,210)=6.24, p<.05]$.

In addition, the three SUC (CC, R, CC+R) and three SIM (CC, R, CC+R) groups were compared. On the TwR measure, no differences or interactions between the SUC groups were observed. However, for the SIM groups, the first-transfer-block TwR performance for the $R$ group was significantly lower than that for either the CC group or the $\mathrm{CC}+\mathrm{R}$ group $[\mathrm{Fs}(1,210)=14,79$ and 4.51 , respectively; ps $<.01$ and $<.05$, respectively]. The SIM CC and CC+R groups did not differ in transfer on the TwR measure. On the DI measure, the SUC CC, $R$, and $C C+R$ groups did not differ after the first block of transfer testing. On the first block, the $\mathrm{CC}$ and $\mathrm{CC}+\mathrm{R}$ groups did not differ, but both had higher DI performances than the SUC R group $[\operatorname{Fs}(1,210)=8.25$ and 4.36 , respectively; ps $<.01$ and $<.05$, respectively]. The SIM groups differed on the DI measure over the first two 3day blocks. For the first block of transfer testing, the CC group was superior to the $\mathrm{CC}+\mathrm{R}$ group, the $\mathrm{CC}+\mathrm{R}$ group was superior to the $R$ group $[\mathrm{Fs}(1,210)=43.64$ and 5.38 , respectively; ps $<.01$ and $<.05$, respectively], and the $C C$ was necessarily superior to the $R$ group $[F(1,210)=$ $79.28, \mathrm{p}<.01]$. On the second block of transfer testing, the SIM CC group was still superior to the CC+R and $R$ groups $[F s(1,210)=11.55$ and 21.47 , respectively; ps $<.01]$, but the $R$ and $C C+R$ groups did not differ. No differences between the SIM CC, R, and CC $+\mathrm{R}$ groups were observed after the second block of transfer testing.

An additional question concerns the level of discriminative performance for these groups in transfer testing. The DI measure allows a .01 chance discrimination envelope to be easily incorporated into the figure. Figure 2 shows that groups with changed colors (CC) continued to discriminate between brightness signals well above chance discrimination levels, although the SIM group is superior to the SUC group. However, for the $\mathrm{R}$ and $\mathrm{CC}+\mathrm{R}$ transfer conditions, discrimination performances of the SIM $\mathrm{R}$ and $\mathrm{CC}+\mathrm{R}$ groups drop to levels significantly below chance, whereas for the SUC R and CC +R groups, DI performances drop to chance levels. A last analysis found no differences between balanced halves with $\mathrm{H}$ or $\mathrm{L}$ brightness signals paired with shock, and no differences between red and green color signals.
Clearly, goldfish trained with the SIM procedure learned faster and had larger positive and negative transfer effects, and, following reversal, relearned faster than goldfish trained with the SUC procedure. One could argue that the differences between the SIM and SUC procedures are not because of their respective successive and simultaneous presentations of choice signals, but rather because of the signals the animal is required to approach (swim toward) when an initial response turns on the choice signal(s). When the first response turns on the shockpaired signal (SUC) or places the goldfish in the shockpaired signal end (SIM), both the SUC and the SIM procedure require an additional response to alternate signals (SUC) or ends (SIM) to avoid shock. But the SUC animal must approach (until it gets to the far side of the top of the hurdle) the shock-paired signal, whereas the SIM animal approaches the safe or shock-omission paired signal. Thus the SUC procedure may produce response inhibition (approach shock-paired signal), but the SIM procedure would not. If the first response turns on the safe signal (SUC) or places the animal on the end showing the safe signal (SIM), an additional response is not only unnecessary to avoid shock, but would place the animal in jeopardy of shock. The probability of the additional response might be less for the SIM procedure, because it would involve approaching the shock-paired signal, whereas the SUC animal would approach the apparently safe signal. Thus, both initial-response-signal conditions would appear to favor the SIM-trained animal, since its response would not be inhibited when it was necessary but would be inhibited when the additional response placed it in jeopardy of shock. But this explanation presumes that, if its responses are to be differentially inhibited by signals at one end of the shuttlebox, the goldfish at the other end (or at least from the top of the hurdle) can see and recognize the signals at both ends. If one can assume that the goldfish is able to see signals at both ends of the shuttlebox, it at least implies the possibility that the two procedures do represent forms of successive and simultaneous presentation and the possibility in the SIM procedure for relational comparison (Mackintosh, 1974). The possibility does not exist in the SUC procedure, simply because both signals are never present at the same time. But, because of the superiority of the SIM over the SUC procedure, both in acquisition and transfer tests, the SIM procedure becomes the procedure of choice in testing goldfish for the transposition effect.

\section{EXPERIMENT 2}

Testing for transposition is, in principle, a fairly easy task. First, one must have available three signals on a common dimension [e.g., low brightness (L), medium brightness $(\mathrm{M})$, and high brightness $(\mathrm{H})]$. Following discrimination training with two of these signals (e.g., L vs. M), the animal is tested in transfer with one signal retained (e.g., M) and the other signal changed (e.g., L changed 
to $\mathrm{H})$. Note that, from original learning to transfer, the brightness relationship of the retained $\mathrm{M}$ signal to the other signal is "reversed" (i.e., $M>L$ to $M<H$ ). If the animal learns to prefer the $M$ (brighter) signal in original learning and continues to do so in transfer testing, it implies that it has learned something "absolute" about the M signal in original learning. However, if the animal chooses the $\mathrm{H}$ (relationally brighter) signal in transfer, transposition has been demonstrated. However, demonstrating the transposition phenomenon does not automatically imply that relational learning has occurred. Other nonrelational learning interpretations of the transposition effect exist (e.g., Spence, 1937). But it is only when transposition can be reliably demonstrated that the mechanisms producing it can be investigated. Since, as far as the author knows, transposition has yet to be demonstrated using negative reinforcement procedures, the ability of goldfish to learn brightness discrimination problems in order to avoid shock and the availability of the SIM presentation procedure for the shuttlebox apparatus seem to provide the ideal opportunity for testing for negatively reinforced transposition.

\section{Subjects \\ Method \\ Forty-eight 5- to 6-cm-long goldfish from the same source as the fish used in Experiment 1 served as subjects. All treatment and main- tenance procedures were identical to those of Experiment 1.}

\section{Apparatus}

The shuttleboxes were the same as in Experiment 1 except for the following. In addition to the top (ITI) light fixture and end (response-contingent) light fixtures, an additional fixture, placed beneath each shuttlebox, cast light up into the apparatus, illuminating the center hurdle through a $5-\mathrm{cm}$ slit in the otherwise flat black bottom. This additional light source was used to indicate trial onset to the goldfish. All light was provided by 7-W 11-V ac Christmas tree bulbs, as before. Response-contingent lights in the end fixtures were green in color $(517 \pm 2 \mathrm{mu})$ at low $(\mathrm{L})$, medium $(\mathrm{M})$, and high (H) brightness levels. A Minolta Spot Meter M was used to measure luminances at each brightness level, as in the previous experiment. Luminances in the four shuttleboxes were found to range from 0.15 to $0.35 \mathrm{~mL}$ for $\mathrm{L}$, from 0.74 to $1.54 \mathrm{~mL}$ for $\mathrm{M}$, and from 5.43 to $6.73 \mathrm{~mL}$ for $\mathrm{H}$ levels. The trial start signals from underneath the center hurdles were red $(620 \pm 4 \mathrm{mu})$ and ranged from 8 to $13 \mathrm{~mL}$. White ITI illumination, provided by overhead fixtures, was identical to that in Experiment 1. All other apparatus features were the same as in the previous experiment.

\section{Procedure}

Experiment 2 was divided into two phases-a 15-day original learning phase and a 5-day transfer testing phase. With the following exceptions, all procedural details were the same as in Experiment 1 .

At the start of each trial, the white overhead ITI light was extinguished and the red light underneath the hurdle turned on, indicating trial onset. The goldfish's first response of the trial extinguished the red light underneath the hurdle and turned on the two end lights. The end lights were controlled exactly as in the SIM procedure of Experiment 1. Each trial lasted $15.2 \mathrm{sec}$, with shock scheduled to occur at 12,14, and $15 \mathrm{sec}$. After $15.2 \mathrm{sec}$, all trial illumination was extinguished, and the overhead white illumination was reestablished.
Table 1

The Signals and Shock Pairing in

Original Learning and Transfer

\begin{tabular}{ccc}
\hline $\begin{array}{c}\text { Original Learning } \\
\text { and }\end{array}$ & $\begin{array}{c}\text { Transfer Signals } \\
\text { and }\end{array}$ & Predictions \\
Shock Pairing & Shock Pairing & "Absolute" "Relational" \\
\hline
\end{tabular}

Brighter Paired

$$
[L+M-](n=12) \quad \begin{array}{llll}
{[M-H+](S)(n=6)} & \text { positive } & \text { negative } \\
{[M+H-](R)(n=6)} & \text { negative } & \text { positive }
\end{array}
$$

Brighter Paired

$$
[M+H-](n=12) \begin{array}{lll}
{[L-M+](S)(n=6)} & \text { positive } & \text { negative } \\
{[L+M-](R)(n=6)} & \text { negative } & \text { positive }
\end{array}
$$

Dimmer Paired

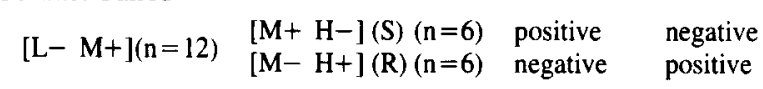

Dimmer Paired

$$
[M-H+](n=12) \quad \begin{array}{llll}
{[L+M-](S)(n=6)} & \text { positive } & \text { negative } \\
{[L-M+](R)(n=6)} & \text { negative } & \text { positive }
\end{array}
$$

Note-The brightness levels of the signals $(L, M$, and $H)$ and shock pairing $(+=$ shock-omission paired, $-=$ shock-paired $)$ for all groups in both original learning and transfer testing, including the number (n) per group. In each row, the two transfer groups generated from the original learning condition have either their retained $M$ signal paired with shock the same $(S)$, as it was in original learning, or the shock pairing reversed $(R)$ from the way it was in original leaming. In addition, transfer performance predictions for "absolute" and "relational" original learning interpretations are shown.

Original learning. In original learning, half $(n=24)$ of the goldfish were trained with $L^{*}$ and $M^{*}$ signals and the remainder $(n=24)$ were trained with $M^{*}$ and $H^{*}$ signals. Half of the $L^{*} M^{*}$ group and half of the $\mathrm{M}^{*} \mathrm{H}^{*}$ signal group had the brighter signal paired with shock $(-)$ and the other signal paired with shock omission $(+)$; the remaining halves of the two groups had the dimmer signal paired with shock and the brighter signal paired with shock omission. This constituted a brightnesses $\times$ shock pairing factorial design, with individual groups $(n=12)$ having $\mathrm{L}+\mathrm{M}-, \mathrm{L}-\mathrm{M}+, \mathrm{M}+\mathrm{H}-$, and $\mathrm{M}-\mathrm{H}+$ paired signals. The signals and shock pairings of these groups are given in the first column of Table 1. Although an erratic equipment problem affected the $\mathbf{M}^{*} \mathbf{H}^{*}$; groups during the first week of training, the problem was solved and the experiment was continued. As will be seen, since the goldfish did learn to discriminate between different brightness levels, the transfer test for transposition was run.

Transfer testing. Each of the four original learning groups had its $\mathrm{L}$ or $\mathrm{H}$ signal replaced in transfer ( $\mathrm{L}$ replaced $\mathrm{H}$ and $\mathrm{H}$ replaced $L)$ while retraining the $M$ signal. Half $(n=6)$ of each original learning group had the shock pairing of their $M$ signals the same (S) in transfer as it was in original learning, and half $(n=6)$ had the shock pairing of their $M$ signals reversed $(R)$ in transfer from that of original learning. This generated eight separate transfer groups, two for each of the four original learning groups. The transfer signals and shock pairings of these eight transfer groups are listed in the second column of Table 1 . Note that Table 1 also lists, in the third column, the transfer performance predictions for each of these transfer groups according to an original-learning "absolute" or "relational" interpretation. Halves $(n=6)$ of each original learning group were equated before transfer testing. All transfer groups, once the appropriate signal and shock pairing changes were accomplished, were tested with the same procedure and against the same criteria as in original learning.

Learning measures. The learning measures were the same as those used in Experiment 1 


\section{Results and Discussion}

The data for the original learning and transfer testing phases were separated to facilitate analysis and presentation. For each phase, the main analyses were four-way ANOVAs, with three between factors and one repeated factor. The between factors were signal brightnesses $\left(L^{*} M^{*}\right.$ vs. $\left.M^{*} H^{*}\right)$, whether the brighter $(L+M-$, $\mathrm{M}+\mathrm{H}-)$ or dimmer $(\mathrm{L}-\mathrm{M}+, \mathrm{M}-\mathrm{H}+)$ signal was paired with shock, and whether the $M$ signal in transfer had the same (S) shock pairing as in original learning or the $M$ signal-shock pairing was reversed $(R)$ between original learning and transfer. This latter $S$ versus $R$ factor was included in the original learning data analysis to determine if the halves of each original learning group were equal before transfer testing. The repeated factor was 15 days of training in the original learning analyses, and 5 days of testing in transfer.

\section{Original Learning}

The same/reversed (S/R) transfer shock pairing factor produced no statistically significant effects in any of the original learning analyses, either as a main effect or in any interactions, which supported the assumption that the halves of the original learning groups were equal before the transfer manipulations. Because the $S / R$ factor had no effects, it was omitted from further consideration or discussion in the original-learning results.

The primary original learning results are shown in Figures 3 and 4 . Figure 3 shows the mean trials with response (TwR) and mean avoidances over days for all

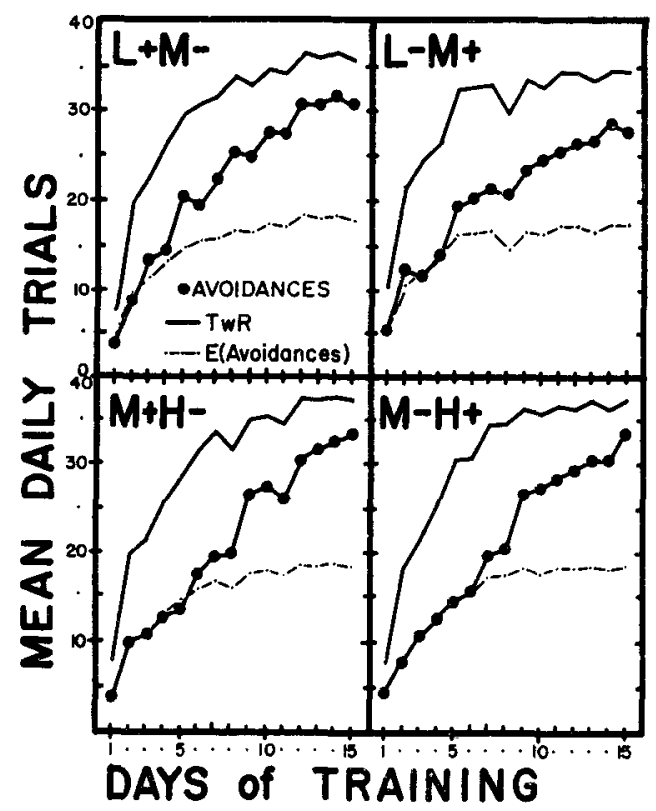

Figure 3. The mean daily trials with response before shock (TwR), mean avoidances, and $\mathbf{E}$ (avoidances) for all four original-learning groups are shown for the 15 days of original learning. E (avoidance), which is $.5 \times T w R$, is included for comparative purposes only. Groups in different panels differ by the brightnesses of their signals $(L, M$, H) and their specific signal-shock pairings $(+,-)$.

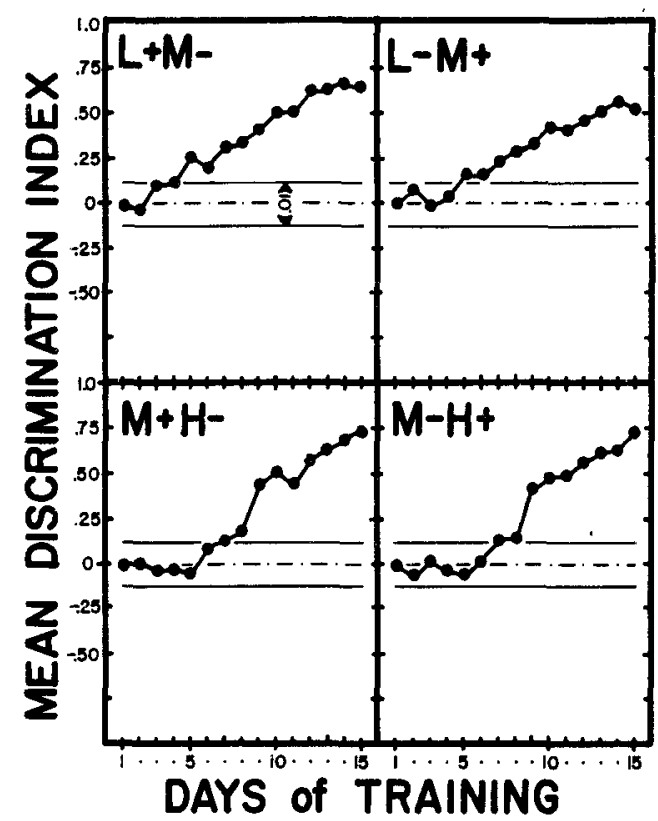

Figure 4. The mean daily discrimination indexes (DI) for the four different groups over the $\mathbf{1 5}$ days of original learning. Groups in different panels differ by the brightnesses of their signals $(L, M$, $H)$ and signal-shock pairings $(+,-)$. In addition, a .01 chance discrimination envelope is incorporated into each panel. DI performances outside of this envelope differ from chance by at least .01.

original-learning groups. In addition, Figure 3 shows E (avoidances) for comparative purposes only. Note that all animals in all groups show an increasing number of TwR and avoidances with training, regardless of their signal brightnesses or the specific shock pairings of their signals. Figure 4 shows the DI means for all groups in original learning. The only peculiar result seen occurs in the first week of original learning. During that week (Days 1 to 5), the two $\mathrm{M}^{*} \mathrm{H}^{*}$ groups do not seem to depart from the chance DI (discimination) levels, as did the $\mathrm{L}^{*} \mathrm{M}^{*}$ groups. This was due to an erratic equipment problem, which was solved by the end of the first week. Once the problem was repaired, the $\mathrm{M}^{*} \mathrm{H}^{*}$ groups learned rapidly and did not differ from the $\mathrm{L}^{*} \mathrm{M}^{*}$ groups over the last week of original learning (Days 11 to 15). Figure 4 clearly shows this problem, and also that no differences remained at the end of original learning. The advantage of the DI measure is that it allows for the incorporation of a chance discrimination envelope into the figure. This allows for immediate visual identification of when discrimination performances exceed chance levels in either the positive $(+)$ or negative $(-)$ direction. All groups in Figure 4 showed significant discrimination (DI) increases with original-learning training.

All of these impressions were confirmed by the statistical analyses. For the TwR measure, only increases with training were observed $[F(14,560)=194.99, p<.01]$. The avoidance and DI measures produced similar patterns of results, as in the first experiment, so only the DI analyses are reported. A significant increase in DI with train- 
ing $[F(14,560)=116.65, p<.01]$ and the equipmentrelated brightnesses $\times$ training interaction $[F(14,560)=$ $5.25, \mathrm{p}<.01]$ were evident, but this interaction had disappeared by the last 5 days of original learning $[F(4,560)$ $=1.40$ ]. It is clear that all animals learned to discriminate between signals differing in brightness, regardless of the specifics of signal brightness or shock pairing.

\section{Transfer Testing}

The primary comparisons for transposition involve testing the pairs of transfer groups associated with each original-learning condition to determine if they show positive or negative transfer. The transfer-performance predictions for each of the eight transfer groups are given in the right column of Table 1 . To accomplish the planned comparisons, overall ANOVAs, with factors as listed earlier, were computed. This was followed by partitions for transfer pairs associated with each of the original learning conditions. The results are shown in Figures 5 and 6 , where each panel represents the two transfer groups generated from each of the original-learning conditions. In each panel, the two transfer groups differ in the pairing of their retained $M$ signal, with one group's $M$ signal having the same (S) shock pairing as it had in original learning and the other group having its M-signal shock pairing reversed $(R)$ from what it was in original learning. Figure 5 shows the mean TwR and mean avoidances over the 5 days of transfer testing for all transfer groups, arranged in $S / R$ pairs associated with each originallearning condition. In addition, Figure 5 shows $\mathrm{E}$ (avoidances) for comparative purposes only. From Figure 5, it is clear that the halves of each originallearning group with their M-signal-shock pairing reversed (R) in transfer continued to avoid at levels comparable to those achieved in original learning, regardless of their original-learning signal brightnesses or shock pairing. In contrast, the halves that had their M signal paired the same (S) in transfer as in original learning suffer substantial negative transfer effects. Pairs of transfer groups generated from the same original-learning condition responded on comparable numbers of trials (TwR), although there are small differences between the different originallearning conditions. The results of the DI measure are shown in Figure 6. The advantage of the DI measure is clearly shown in Figure 6, where all of the reversed (R) pairing groups show positive transfer (continue to discriminate well above chance levels) whereas all of the same (S) pairing groups show significant negative transfer (discrimination drops to levels significantly below chance) on the first day of transfer testing. It is also clear that each of the same (S) pairing groups relearn the discrimination by the end of the 5 days of transfer testing, to levels well above chance discrimination. Again, all of these impressions were confirmed by statistical analysis.

For the overall TwR measures analysis, slight, but significant, differences between same and reversed signal groups $[F(1,40)=9.80, p<.01]$ and slight, but significant, increases with training $[F(4,160)=4.51, p<.01]$ were observed, but partitions and comparisons of the $S / R$

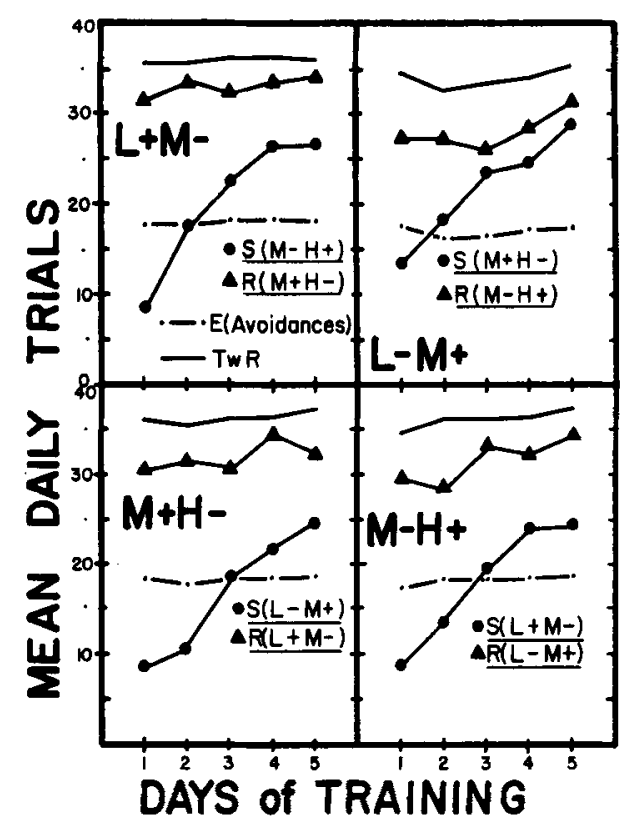

Figure 5. The mean daily trials with response before shock (TwR), mean avoidances, and $\mathbf{E}$ (avoidances) for all eight groups tested for 5 days in transfer. Each panel shows the two transfer groups generated from the designated original-learning condition. One group has its $M$ signal paired the same (S) as it was in original learning, and the other has the shock pairing of its $M$ signal reversed $(R)$ in transfer. The $E$ (avoidance) measure is presented for comparative purposes only.

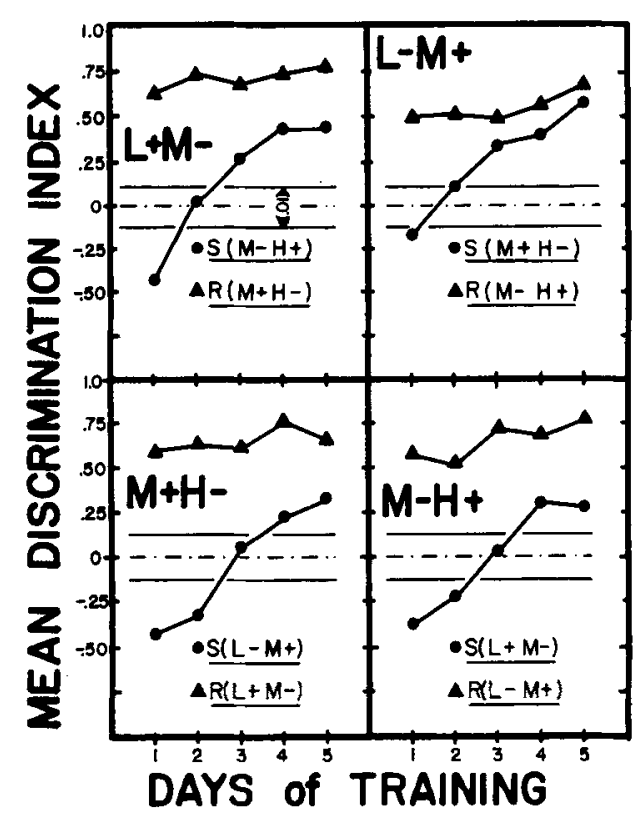

Figure 6. The mean daily discrimination indexes (DI) for all eight transfer groups over the 5 days of transfer testing. Each panel represents the two transfer groups generated from the designated original-learning condition. One group has its $M$ signal paired the same (S) as it was in original learning, and the other has the shock pairing of its $M$ signal reversed $(R)$ in transfer. In addition, a .01 chance discrimination envelope is incorporated into each panel. DI performances outside of this envelope, regardless of direction, indicate discrimination levels differing from chance. 
pairs associated with each original-learning group found no differences between them. Because of this, only one TwR measure is shown per panel. For the DI measures, the main analysis found $\mathrm{R}$ groups significantly better than $S$ groups $[F(1,40)=110.70, p<.01]$, significant increases in DI over transfer testing $[F(4,160)=61.16$, $\mathrm{p}<.01]$, and, as expected, a significant $\mathrm{S} / \mathrm{R} \times$ training interaction $[F(4,160)=26.92, p<.01]$. Partitions of the main analysis to compare individual $S / R$ pairs found $\mathrm{R}$ superior to $\mathrm{S}$ for all original-learning conditions $[\operatorname{Fs}(1,40)=30.98,8.24,34.10$, and 27.28 , respectively, for the $\mathrm{L}+\mathrm{M}-, \mathrm{L}-\mathrm{M}+, \mathrm{M}+\mathrm{H}-$, and $\mathrm{M}-\mathrm{H}+$ originallearning conditions; all ps $<.01$ ]. In addition, all partitions revealed significant increases in DI $[F s(4,160)=$ $13.81,12.95,13.77$, and 17.34, respectively; all ps < $.01]$ and interactions between days of transfer training and $\mathrm{S} / \mathrm{R}$ conditions $[\mathrm{Fs}(4,160)=11.50,4.55,10.13$, and 5.98 , respectively; $\mathrm{ps}<.01]$. The $\mathrm{R}$ groups did not differ from each other $[F(3,40)=1.61]$ and the $S$ groups did not differ from each other $[F(3,40)=2.07]$, regardless of their original-learning group identification. The $\mathbf{R}$ groups showed slight, but significant, increases in DI over transfer testing $[\mathrm{F}(4,160)=4.11, \mathrm{p}<.01]$, whereas the $S$ groups showed massive DI increases with transfer training $[F(4,160)=83.97, p<.01]$. The nature of the interactions for each partition is clearly obvious in Figure 6. The $\mathbf{R}$ halves of each original-learning group continued to discriminate at levels comparable to their originallearning performances, whereas each $S$ half suffered significant and massive negative transfer effects and discriminated at levels significantly below chance on the first day of transfer testing. However, all $S$ groups relearned the discrimination with additional transfer testing. The same pattern of results was found for the avoidances.

\section{GENERAL DISCUSSION}

The present results extend what is known about the avoidance-learning abilities of goldfish in the shuttlebox apparatus. In the first experiment, goldfish trained with either the SIM or the SUC procedure learned to avoid shock by choosing between same-color signals differing only in brightness, but SIM training yielded faster learning and produced higher asymptotes than SUC training. Furthermore, SIM training yielded more positive transfer when signal colors were changed $(\mathrm{CC})$, more negative transfer when brightness-level-shock pairing was reversed $(R)$, and faster relearning with signal-shock pairing reversed than did SUC training. When both signal colors and shock pairing were changed $(\mathrm{CC}+\mathrm{R})$, although transfer was attenuated, SIM animals still showed larger negative transfer effects than did SUC animals. Furthermore, all effects were independent of the brightness level paired with shock.

There are at least two ways to explain the superiority of the SIM procedure over the SUC procedure. The first, and most intriguing, is that goldfish learn a relationship between signal brightness and shock pairing in the SIM procedure, but not in the SUC procedure. Presumably, learning the brightness-level-shock pairing relationship might be possible in the SIM procedure because both signals are present in the apparatus at the same time, whereas in the SUC procedure relational learning would be prohibited because the signals are presented one at a time and never together. This is exactly the same explanation used to account for the superiority of simultaneous over successive presentation procedures in the acquisition and transfer of discriminations based on positive reinforcement techniques (see Mackintosh, 1974). However, because both the SUC and SIM procedures are shock reinforced, a differential inhibition explanation is possible, based only on the assumption that signals paired with shock inhibit approach responses and signals paired with shock-omission do not. Specifically, if the initial response of a trial produces the shock-paired signal, both ends of the apparatus show the same signal for the SUC animal, but, for the SIM animal, the end occupied shows the shock-paired signal and the other end shows the shockomission signal. Although an additional response is required to avoid shock in both procedures, the SUC animal must approach the shock-paired signal on the other end, whereas the SIM animal approaches the safe (shockomission paired) signal. If the initial response produces the safe signal, no further response is necessary to avoid shock in either the SUC or the SIM procedure. In fact, an additional response puts the animal in jeopardy of shock. The probability of an additional response may be less for the SIM animal than for the SUC animal because the SIM animal would have to approach the shock-paired signal on the other end, whereas the SUC animal would approach an apparently safe signal. Essentially, the response inhibition gradients would favor the SIM over the SUC procedure because, by comparison, the SUC animal would have its shuttling response inhibited when the additional response was necessary to avoid shock but not inhibited when the additional response was not only unnecessary, but jeopardizing. But the response-inhibition explanation still assumes that the "other end" must be visible from the end ocupied, or at least from the hurdle top, which implies the possibility of the visual simultaneous comparison of both ends and signals in the SIM procedure. But, regardless of the mechanism of difference, the superiority of SIM over SUC in both acquisition and transfer tests clearly identifies SIM as the procedure of choice in any attempt to demonstrate shockreinforced transposition.

In Experiment 2, goldfish learned to choose between signals differing in brightness, and then showed both the positive and negative aspects of the transposition phenomenon in the appropriate transfer tests. For instance, when the shock pairing of the signal retained in transfer was changed (reversed) in transfer testing (e.g., $\mathrm{L}+\mathrm{M}-$ to $\mathrm{M}+\mathrm{H}-$ ), goldfish continued to avoid shock by choosing the relationally dimmer $M+$ signal, despite the fact that $M$ had been paired with shock in acquisition. When the shock pairing of the retained signal was identi- 
cal in acquisition and transfer testing (e.g., $\mathrm{L}+\mathrm{M}-$ to $\mathrm{M}-\mathrm{H}+$ ), goldfish avoidance performance dropped to below chance levels in a way similar to what was observed in Experiment 1 following the reversal of signal shock pairing in transfer testing. Furthermore, positive and negative aspects of the transfer phenomenon were independent of the brightness levels paired with shock or shock omission in acquisition, as is clearly shown in Figures 5 and 6 . Clearly, goldfish, after learning to discriminate between signals differing in brightness, show all aspects of the transposition phenomenon when trained with negative or shock reinforcement procedures.

There are at least two ways to interpret the present transposition results. One can assume that the goldfish learned the shock-pairing brightness relationship in acquisition and responded according to that relationship in the transfer test, or that the mechanism of transfer did not involve any relational learning but was the result of something like the summation of positive and negative generalization gradients a la Spence (1937). But elucidating the mechanism of negatively reinforced transposition was not the aim of the second experiment. Transposition, to the author's knowledge, has never been demonstrated in a negative reinforcement paradigm prior to the present demonstration. Now that procedures exist for producing negatively reinforced transposition, work investigating the underlying mechanisms and comparing them with the mechanisms of positively reinforced transposition can begin.

\section{REFERENCES}

Bitterman, M. E., Tyler, D. W.. \& Elam, C. B. (1955). Simultaneous and successive discrimination under identical stimulating conditions. American Journal of Psychology, 68, 237-248.

Gonzales, R. C., \& ShePP, B. E. (1961). Simultaneous and successive discrimination-reversal in the rat. American Journal of Psychologv, 74, 584-589.

KoHLER, W. (1939). Simple structural functions in the chicken and in the chimpanzee. In W. D. Ellis (Ed.), A source book of Gestalt psychology (pp. 217-227). New York: Harcourt, Brace. (Original work published 1918)
LAWrenCE. D. H., \& DeRivera, J. ( 1954). Evidence for relational transposition. Journal of comparative and Physiological Psychology, 47. 465-471.

MacCaslin. E. F. (1954). Successive and simultaneous discrimination as a function of stimulus similarity. American Journal of Psychologv, 67, 308-314.

MaCkINTOSh, N. J. (1974). The psychology of animal learning. London: Academic Press.

MARSH, G. (1967). Relational learning in the pigeon. Journal of Comparative and Phvsiological Psvchologv, 64, 519-521.

Perkins, F. T. (1931). A further study of configurational learning in the goldfish. Journal of Experimental Psychology, 14, 508-538.

Perkins, F. T., \& Wheeler, R. H. (1930). Configurational learning in the goldfish. Comparative Psychologycal Monographs, 7, 1-50.

RIIEY. D. A. (1958). The nature of the effective stimulus in animal discrimination learning: Transposition reconsidered. Psychological Review, 65, 1-7.

Riley, D. A. (1968). Discrimination learning. Boston: Allyn \& Bacon.

SACHS, L. B. (1969). Effects of stimulus comparison during discrimination training on subsequent transposition and generalization gradients. Psychonomic Science, 14, 247-248.

SPENCE, K. W. (1937). The differential response in animals to stimuli varying within a single dimension. Psychological Review, 44, 430-444.

SPENCE, K. W. (1952). The nature of the response in discrimination learning. Psvchological Review, 59, 89-93.

ZERBoLIO, D. J., JR. (1981). Discriminated avoidance learning and reversal by goldfish in a shuttlebox using a linear presentation procedure. Animal Learning \& Behavior. 9, 346-356.

Zerbolio. D. J. \& \& Royalty, J. L. (1982). Acquisition and extinction of differential responses to signals paired with shock or shock-omission in goldfish: Evidence for truly discriminated avoidance learning. Animal Learning \& Behavior, 10. 377-389.

Zerbolio, D. J., \& Royalty, J. L. (1983). Matching and oddity conditional discrimination in the goldfish as avoidance responses: Evidence for conceptual avoidance learning. Animal Learning \& Behavior. 11. 341-348.

Zerbolio, D. J., JR., \& Wickstra, L. L. (1979). Instrumentally based conditioned avoidance response acquisition in goldfish in a simultaneous presentation task. Bulletin of the Psychonomic Society, 13. 311-313.

ZeRbOLIO, D. J., JR., \& WICKSTRA, L. L. (1980). Instrumental avoidance acquisition by goldfish in a $Y$-maze using explicit and responsecontingent cues. Animal Learning \& Behavior. 9, 304-310.

(Manuscript received August 23, 1984: revision accepted for publication February 19, 1985.) 